Supplement Issue 1: International Tehran Breast Cancer Congress (TBCC9)

\title{
Investigation KIT Gene Expression in Sporadic Breast Cancer Tissues
}

\author{
Maryam Rahimi ${ }^{1}$, Farkhondeh Behjati ${ }^{1}$, Fatemeh Moghaddam ${ }^{1}$, \\ Elahe Keyhani ${ }^{1, *}$ \\ ${ }^{1}$ Genetics Research Center, University of Social Welfare and Rehabilitation Sciences, \\ Tehran, Iran \\ ${ }^{*}$ Corresponding Author: Elahe Keyhani, Genetics Research Center, University of \\ Social Welfare and Rehabilitation Sciences, Tehran, Iran.E-mail: ekeyhani1058@ \\ gmail.com
}

DOI: $10.21859 /$ mci-supp-63

\section{Keywords:}

KIT Gene

Overexpression

Breast Cancer

\begin{abstract}
Introduction: Cancer is one the important factor of mortality in the world. Breast cancer is particularly prevalent among women. KIT gene, the primary factor of cell differentiation, proliferation and angiogenesis pathways, which its relation to different cancers has been proved. This gene expression increases in gastrointestinal cancers (90\%), leukaemia (68\%), mast cell tumors (70\%), as well as melanoma. Previous studies has revealed that c-kit is expressed in the normal breast ductal epithelium as well as myoepithelial cells. Furthermore an increased expression in malignant tumors occurs. In this study we analyzed KIT gene expression level in patients suffering from sporadic breast cancer.

Materials and Methods: 48 female breast cancer patients were investigated to determinate KIT gene expression level using IHC method.

Results: 27 out of 48 patients tissues' (56\%) had increased of KIT gene expression in comparison with normal tissues, as a negative control.

Conclusions: Current study, similar to previous studies, provides the evidences that KIT gene expression level increases in tissues of breast cancer and the overexpression of this gene may be a risk factor for development of metastasis of sporadic breast cancer. KIT regulates apoptosis, cell differentiation, proliferation, and angiogenesis which could be an important factor in cancer development and increased risk of developing breast cancer. So this gene can be used for targeted therapy with tyrosine kinase inhibitors which have been successfully administrated in various cancers. Further studies with more detailed data are needed to verify these initial findings.
\end{abstract}

